International Mathematical Forum, 1, 2006, no. 23, 1099 - 1112

\title{
3-Tuple domination number in complete grid graphs
}

\author{
Javad Mehri and MirKamal Mirnia \\ Department of Mathematical Sciences \\ Tabriz University, Tabriz, Iran \\ JMehri@TabrizU.AC.IR \\ S.M. Sheikholeslami \\ Department of Mathematics \\ Azarbaijan University of Tarbiat Moallem, Tabriz, Iran
}

\begin{abstract}
In a graph $G$, a vertex dominates itself and its neighbors. A subset $S \subseteq V(G)$ is a $k$-tuple dominating set of $G$ if each vertex of $G$ is dominated by at least $k$ vertices of $S$. A $k$-tuple dominating set $S$ of a graph $G$ is perfect if each vertex of $G$ is dominated by exactly $k$ vertices in $S$. The $k$-tuple domination number $\gamma_{\times k}(G)$ is the minimum cardinality among all $k$-tuple dominating sets of $G$. In this note we determine the 3 -tuple domination number $\gamma_{\times 3}$ for the complete grid graphs $P_{n} \times P_{m}$ for $2 \leq n \leq 4$ and $m \geq 1$. We also study the existence and construction of perfect 3 -tuple dominating sets in this graphs.
\end{abstract}

Keywords: complete grid graphs, domination number.

Mathematics Subject Classification: 05C69

\section{Introduction}

Let $G$ be a graph with vertex set $V$. A vertex $v \in V$ is said to dominate all the vertices in its closed neighborhood $N[v]$. (For graph theory we follow the notation and terminology of [4].) A subset $S$ of $V$ is a $k$-tuple dominating set of $G$ if each vertex of $G$ is dominated by at least $k$ vertices in $S$ (see [5]). When $k=1,2$ the set $S$ is called dominating set and double dominating set in $G$, respectively(see [5], [6]). When each vertex of $G$ is dominated by exactly $k$ element of $S$, then $S$ is called a perfect $k$-tuple dominating set of $G$. The $k$ tuple domination number $\gamma_{\times k}(G)$ is the minimum cardinality among all $k$-tuple 
dominating sets of $G$. A minimum $k$-tuple dominating set of $G$ is a $k$-tuple dominating set of cardinality $\gamma_{\times k}(G)$. The concept of domination in graph theory is a suitable model for many locating problems in operations research [10]. Dominating sets in graphs have been studied in the past $([2,3,8])$. Perfect dominating sets and Perfect double dominating sets for certain graphs have also been investigated $[1,7,11,9]$. In this paper we focus on 3 -tuple domination number for the complete grid graphs $P_{n} \times P_{m}$ for $2 \leq n \leq 4$ and $m \geq 1$ and study the existence and construction of perfect 3 -tuple dominating sets for grids, cylinders and torus. The paper is organized as follows: In the section 2 we determine 3 -tuple domination number for the complete grid graphs $P_{n} \times P_{m}$ for $2 \leq n \leq 4$ and $m \geq 1$. In Section 3 we completely characterize all grids, cylinders and torus that possess a Perfect 3-tuple domination set and also specify the structure of the existing Perfect 3-tuple domination set.

\section{3-tuple domination numbers for $P_{n} \times P_{m}$}

Throughout this section $\mathfrak{T}$ is a 3 -tuple dominating set in $P_{n} \times P_{m}$. We assume that the vertices of the $i$ th copy of $P_{n}$ in $P_{n} \times P_{m}$ are $u_{1}^{i}, u_{2}^{i}, \ldots, u_{n}^{i}$ for $i=$ $1,2, \ldots, m$. (See Figure 1.)

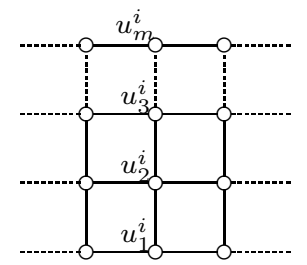

Figure 1: The $i$ th copy of $P_{n}$ in $P_{n} \times P_{m}$

Definition 1 A sequence $\left(c_{1}, c_{2}, \ldots, c_{m}\right)$ of nonnegative integers will be called a column triple domination sequence or simply triple domination sequence for $P_{n} \times P_{m}$ if there is a triple dominating set $\mathfrak{T}$ for $P_{n} \times P_{m}$ such that

$$
c_{j}=\left|\mathcal{C}_{j}\right| \quad \text { for } j=1,2, \ldots, m, \quad \text { where } \mathcal{C}_{j}=\mathfrak{T} \cap C_{j} .
$$

The sequence $\left(\mathcal{C}_{1}, \mathcal{C}_{2}, \ldots, \mathcal{C}_{m}\right)$ is called (column) triple dominating sets sequence.

Similarly, one can define row triple domination sequence and row triple dominating sets sequence.

Clearly $\gamma_{\times 3}\left(P_{n} \times P_{m}\right)=\gamma_{\times 3}\left(P_{m} \times P_{n}\right)$ and $\gamma_{\times 3}\left(P_{n} \times P_{m}\right)$ is the minimum of $c_{1}+c_{2}+\ldots+c_{m}$ over all triple domination sequences $\left(c_{1}, c_{2}, \ldots, c_{m}\right)$ for $P_{n} \times P_{m}$. 
Every vertex in $C_{1}$ and $C_{m}$ is dominated exactly by one vertex of $C_{2}$ and $C_{m-1}$, respectively. So, The set $\mathcal{C}_{1}$ is a double dominating set for $C_{1}$, and also $\mathcal{C}_{m}$ for $C_{m}$. Hence, we have

Proposition 2 For every $m, n \geq 1, c_{1} \geq\left\lceil\frac{2 n+2}{3}\right\rceil$ and $c_{m} \geq\left\lceil\frac{2 n+2}{3}\right\rceil$.

Every vertex in $C_{i}, i=2,3, \ldots, m-1$ is dominated exactly by two vertices of $V\left(P_{n} \times P_{m}\right) \backslash C_{i}$. So, The set $\mathcal{C}_{i}$ is a dominating set for $C_{i}$, and we have the following two results

Proposition 3 For every $m, n \geq 1$, and for all $i=2,3, \ldots, m-1, c_{i} \geq\left\lceil\frac{n}{3}\right\rceil$.

Proposition 4 For some $i \in\{2,3, \ldots, m-1\}, c_{i}=\frac{n}{3}$ if and only if $c_{i-1}=$ $c_{i+1}=n$.

Every vertex in $C_{i}$ or $C_{i+1}, i=2,3, \ldots, m-2$ is dominated only by one vertex of $V\left(P_{n} \times P_{m}\right) \backslash\left\{C_{i} \cup C_{i+1}\right\}$. So, The set $\mathcal{C}_{i} \cup \mathcal{C}_{i+1}$ is a double dominating set for $C_{i} \cup C+i+1$, and we have

Proposition 5 For every $m, n \geq 1$, and for all $i=2,3, \ldots, m-2, c_{i}+c_{i+1} \geq$ $n+1$.

\section{$2.1 \quad 3$-tuple domination numbers for $P_{2} \times P_{m}$}

Theorem 6 For all $m \geq 3, \gamma_{\times 3}\left(P_{2} \times P_{m}\right)=\left\lceil\frac{3(m+1)}{2}\right\rceil$.

Proof. Let $\mathfrak{T}$ be a 3-tuple dominating set in $P_{2} \times P_{m}$. Since $u_{1}^{1}, u_{2}^{1}$ must be dominated by at least three vertices of $\mathfrak{T}$ we must have $c_{1}=c_{2}=2$. Similarly, $c_{n}=c_{n-1}=2$. By Proposition 5 we have $c_{i}+c_{i+1} \geq 3$ for $i=2,3, \ldots, m-2$. Now, we have

$$
2|\mathfrak{T}|=\sum_{1}^{m} 2 c_{i}=\left(2 c_{1}+c_{2}\right)+\sum_{2}^{m-2}\left(c_{i}+c_{i+1}\right)+\left(c_{m-1}+2 c_{m}\right) \geq 3(m+1) .
$$

Thus we have $|\mathfrak{T}| \geq\left\lceil\frac{3(m+1)}{2}\right\rceil$.

Now we define a 3 -tuple dominating set in $P_{2} \times P_{m}$ with $\left\lceil\frac{3(m+1)}{2}\right\rceil$ vertices. Define

$$
\begin{aligned}
\mathfrak{T}= & \left\{u_{1}^{i}, u_{2}^{i} \mid i=1, m-1, m, 2 k \text { and } k=1,2, \ldots,\left\lfloor\frac{m}{2}\right\rfloor\right\} \cup \\
& \left\{u_{1}^{i} \mid i=4 k+3, k=0,1, \ldots,\left\lfloor\frac{m}{4}\right\rfloor-1\right\} \cup \\
& \begin{cases}\left\{u_{2}^{i} \mid i=4 k+1, k=1, \ldots,\left\lfloor\frac{m}{4}\right\rfloor-1\right\} & \text { if } m \text { is even } \\
\left\{u_{2}^{i} \mid i=4 k+1, k=1, \ldots,\left\lfloor\frac{m}{4}\right\rfloor\right\} & \text { if } m \text { is odd. }\end{cases}
\end{aligned}
$$

Then $\mathfrak{T}$ is a 3 -tuple dominating set in $P_{2} \times P_{m}$ with $\left\lceil\frac{3(m+1)}{2}\right\rceil$ vertices. This completes the proof. $\diamond$ 


\subsection{3-tuple domination numbers for $P_{3} \times P_{m}$}

The proof of the following result is easy and we leave it for the reader.

Lemma $7 \quad 3 c_{1}+c_{2} \geq 11$ and $3 c_{n}+c_{n-1} \geq 11$.

Lemma 8 If $c_{i}=2$ for some $2 \leq i \leq m-2$, then $c_{i}+2 c_{i+1}+c_{i+2} \geq 9$.

Proof. We consider three cases.

case $1 u_{1}^{i}, u_{3}^{i} \in \mathfrak{T}$. This forces $u_{1}^{i+1}, u_{3}^{i+1} \in \mathfrak{T}$, since $u_{1}^{i}$ and $u_{3}^{i}$ must be dominated by at least three vertices of $\mathfrak{T}$. If $c_{i+1}=2$, then we must have $c_{i+2}=3$ which implies $c_{i}+2 c_{i+1}+c_{i+2} \geq 9$. Let $c_{i+1}=3$. By proposition 3 we have $c_{i+2} \geq 1$ which implies $c_{i}+2 c_{i+1}+c_{i+2} \geq 9$.

case $2 \quad u_{1}^{i}, u_{2}^{i} \in \mathfrak{T}$. This forces $u_{2}^{i+1}, u_{3}^{i+1}, u_{3}^{i+2} \in \mathfrak{T}$, since $u_{3}^{i+1}$ must be dominated by at least three vertices of $\mathfrak{T}$. If $c_{i+1}=3$ then obviously $c_{i}+2 c_{i+1}+$ $c_{i+2} \geq 9$. Let $c_{i+1}=2$. This forces $c_{i+2}=3$ which implies $c_{i}+2 c_{i+1}+c_{i+2} \geq 9$. case $3 u_{2}^{i}, u_{3}^{i} \in \mathfrak{T}$. An argument similar to that described in case two shows that $c_{i}+2 c_{i+1}+c_{i+2} \geq 9$. This completes the proof. $\diamond$

Lemma 9 If $c_{i}+2 c_{i+1}+c_{i+2}=8$ for some $1 \leq i \leq m-2$, then either $c_{i+1}+2 c_{i+2}+c_{i+3} \geq 10$ or $c_{i+1}+2 c_{i+2}+c_{i+3}=9$ and $c_{i+2}+2 c_{i+3}+c_{i+4} \geq 10$ or $c_{j}+2 c_{j+1}+c_{j+2}=9$ for $j=i+1, i+2$ and $c_{i+3}+2 c_{i+4}+c_{i+5} \geq 10$ or $c_{j}+2 c_{j+1}+c_{j+2} \geq 9$ for $j=i+1, i+2, i+3, i+4$.

Proof. Obviously, $c_{i+1}<3$. By proposition 4 and lemma 8, we must have $c_{i}=$ 3. We claim that $c_{i+2}=3$. $c_{i+2} \neq 2$, since $2 c_{i+1} \neq 3$. If $c_{i+2}=1$ then $c_{i+1}=3$ by proposition 4 . This implies $c_{i}+2 c_{i+1}+c_{i+2}=10$ which is a contradiction. Thus $c_{i+2}=3$. This forces $c_{i+1}=1$ and $u_{2}^{i+1} \in \mathfrak{T}$. Now, we must have $u_{1}^{i+3}, u_{3}^{i+3} \in \mathfrak{T}$. So $c_{i+3} \geq 2$. If $c_{i+3}=3$ then obviously $c_{i+1}+2 c_{i+2}+c_{i+3}=10$. Let $c_{i+3}=2$. Then $c_{i+1}+2 c_{i+2}+c_{i+3}=9$ and we must have $u_{1}^{i+4}, u_{3}^{i+4} \in \mathfrak{T}$. So $c_{i+4} \geq 2$. If $c_{i+4}=3$, then obviously $c_{i+2}+2 c_{i+3}+c_{i+4}=10$. Let $c_{i+4}=2$. Then $c_{i+2}+2 c_{i+3}+c_{i+4}=9$ and we must have $c_{i+5}=3$ and $c_{i+3}+2 c_{i+4}+c_{i+5}=9$. Now it is easy to see that $c_{i+4}+2 c_{i+5}+c_{i+6} \geq 9$. This completes the proof. $\diamond$

Theorem 10 For every $m>1, \gamma_{\times 3}\left(P_{3} \times P_{m}\right)=\left\lceil\frac{9 m-\left\lceil\frac{m-2}{5}\right\rceil+4}{4}\right\rceil$.

Proof. We have

$$
\begin{aligned}
4|\mathfrak{T}| & =\left(3 c_{1}+c_{2}\right)+\sum_{i=1}^{m-2}\left(c_{i}+2 c_{i+1}+c_{i+2}\right)+\left(3 c_{m}+c_{m-1}\right) \\
& \geq 22+8\left\lceil\frac{m-2}{5}\right\rceil+9\left(m-2-\left\lceil\frac{m-2}{5}\right\rceil\right) \\
& \geq 9 m-\left\lceil\frac{m-2}{5}\right\rceil+4
\end{aligned}
$$

Since By lemma 9 at most one of the consecutive five terms $c_{i}+2 c_{i+1}+c_{i+2}$ is 8 and the other is grater than 8 . Thus $\gamma_{\times 3}\left(P_{3} \times P_{m}\right) \geq\left\lceil\frac{9 m-\left\lceil\frac{m-2}{5}\right\rceil+4}{4}\right\rceil$. 
Now we define a 3 -tuple dominating set of order $\left\lceil\frac{9 m-\left\lceil\frac{m-2}{5}\right\rceil+4}{4}\right\rceil$. Let $m=$ $5 s+r, s, r \in \mathbb{Z}$ and $0 \leq r<m$. Define

$$
\begin{aligned}
\mathfrak{T} & =\left\{u_{i}^{5 k+1}, u_{i}^{5 k+4} \mid 0 \leq k \leq s-1,1 \leq i \leq 3\right\} \cup \\
& \left\{u_{i}^{5 k+2}, u_{i}^{5 k+3}, u_{2}^{5 l+5} \mid 0 \leq k \leq s-1, i=1,3,0 \leq l \leq s-2\right\} \cup \\
& \left\{\begin{array}{ll}
\left\{u_{1}^{m}, u_{2}^{m}, u_{3}^{m}\right\} & \text { if } s \geq 1, r=0 \\
\left\{u_{1}^{m-1}, u_{3}^{m-1}, u_{1}^{m}, u_{2}^{m}, u_{3}^{m}\right\} & \text { if } s \geq 1, r=1 \\
\left\{u_{2}^{m-2}, u_{i}^{m-1}, u_{i}^{m} \mid 0 \leq i \leq 3\right\} & \text { if } r=2 \\
\left\{u_{2}^{m-3}, u_{1}^{m-1}, u_{3}^{m-1}, u_{i}^{m-2}, u_{i}^{m} \mid 0 \leq i \leq 3\right\} & \text { if } r=3 \\
\left\{u_{2}^{m-4}, u_{j}^{m-1}, u_{j}^{m-2}, u_{i}^{m-3}, u_{i}^{m} \mid 0 \leq i \leq 3, j=1,2\right\} & \text { if } r=4 .
\end{array}\right\}
\end{aligned}
$$

It is easy to see that in each case $\mathfrak{T}$ is a 3 -tuple dominating set in $P_{3} \times P_{m}$ with $\left\lceil\frac{9 m-\left\lceil\frac{m-2}{5}\right\rceil+4}{4}\right\rceil$ vertices. This completes the proof. $\diamond$

\subsection{3-tuple domination numbers for $P_{4} \times P_{m}$}

Lemma $11 c_{i}+2 c_{i+1}+c_{i+2} \geq 11$ for $1 \leq i \leq m-2$.

Proof. By proposition 3, $c_{i} \geq 2$ for each $i$. We consider three cases.

case $1 \quad c_{i}=4$. If $c_{i+1}=2$ then we have $c_{i+2} \geq 3$ by proposition 5 . Then $c_{i}+2 c_{i+1}+c_{i+2} \geq 11$. Let $c_{i+1} \geq 3$. Then $c_{i}+2 c_{i+1}+c_{i+2} \geq 11$, since $c_{i+2} \geq 2$. case $2 \quad c_{i}=3$. We consider four subcases.

subcase $1 \quad u_{1}^{i}, u_{2}^{i}, u_{3}^{i} \in \mathfrak{T}$. Since $u_{1}^{i+1}, u_{4}^{i+1}$ must be dominated by three vertices of $\mathfrak{T}$ we must have $u_{3}^{i+1}, u_{4}^{i+1} \in \mathfrak{T}$ and either $u_{1}^{i+1}$ or $u_{2}^{i+1} \in \mathfrak{T}$. Therefore, $c_{i+1} \geq 3$ and also $c_{i+2} \geq 2$ by proposition 3 . This implies $c_{i}+2 c_{i+1}+c_{i+2} \geq 11$. subcase $2 u_{1}^{i}, u_{2}^{i}, u_{4}^{i} \in \mathfrak{T}$. If $c_{i+1} \geq 3$ then as above we have $c_{i}+2 c_{i+1}+c_{i+2} \geq$ 11. Let $c_{i+1}=2$. Since $u_{4}^{i}$ must be dominated by three vertices of $\mathfrak{T}$ we must have $u_{4}^{i+1} \in \mathfrak{T}$. Since also $u_{3}^{i+1}$ must be dominated by three vertices of $\mathfrak{T}$ we must have either $u_{2}^{i+1}$ or $u_{3}^{i+1} \in \mathfrak{T}$. If $u_{2}^{i+1} \in \mathfrak{T}$ then we must have $c_{i+2}=4$ which implies $c_{i}+2 c_{i+1}+c_{i+2} \geq 11$. If $u_{3}^{i+1} \in \mathfrak{T}$ then $u_{1}^{i+1}$ is dominated by at most two vertices of $\mathfrak{T}$ which is a contradiction.

subcase $3 u_{1}^{i}, u_{3}^{i}, u_{4}^{i} \in \mathfrak{T}$. An argument similar to that described in subcase 2 prove that $c_{i}+2 c_{i+1}+c_{i+2} \geq 11$.

subcase $4 u_{2}^{i}, u_{3}^{i}, u_{4}^{i} \in \mathfrak{T}$. As subcase 1 we can see $c_{i}+2 c_{i+1}+c_{i+2} \geq 11$.

case $3 \quad c_{i}=2$. We consider six subcases.

subcase $1 u_{1}^{i}, u_{4}^{i} \in \mathfrak{T}$. This forces $c_{i+1}=4$ and we have $c_{i}+2 c_{i+1}+c_{i+2} \geq 11$, since $c_{i+2} \geq 2$.

subcase $2 u_{1}^{i}, u_{3}^{i} \in \mathfrak{T}$. This forces $u_{1}^{i+1}, u_{3}^{i+1}, u_{4}^{i+1} \in \mathfrak{T}$. If $c_{i+1}=4$ then obviously $c_{i}+2 c_{i+1}+c_{i+2} \geq 11$. Let $c_{i+1}=3$. This forces $u_{1}^{i+2}, u_{2}^{i+2}, u_{4}^{i+2} \in \mathfrak{T}$ which implies $c_{i}+2 c_{i+1}+c_{i+2} \geq 11$. 
subcase $3 u_{1}^{i}, u_{2}^{i} \in \mathfrak{T}$. This case is impossible since then $u_{4}^{i}$ is dominated by at most two vertices of $\mathfrak{T}$ which is a contradiction.

subcase $4 u_{2}^{i}, u_{3}^{i} \in \mathfrak{T}$. Since $u_{1}^{i+1}$ and $u_{4}^{i+1}$ must be dominated by three vertices of $\mathfrak{T}$, we must have $c_{i+1}=4$ which implies $c_{i}+2 c_{i+1}+c_{i+2} \geq 11$.

subcase $5 \quad u_{2}^{i}, u_{4}^{i} \in \mathfrak{T}$. As subcase 2 we have $c_{i}+2 c_{i+1}+c_{i+2} \geq 11$.

subcase $6 \quad u_{3}^{i}, u_{4}^{i} \in \mathfrak{T}$. This case is impossible (see subcase 3 ). This completes the proof. $\diamond$

Lemma 12 If $c_{2}+2 c_{3}+c_{4}=11$ then $4 c_{1}+3 c_{2}+c_{3} \geq 27$. Similarly, If $c_{n-2}+2 c_{n-1}+c_{n}=11$ then $4 c_{n}+3 c_{n-1}+c_{n-2} \geq 27$.

Proof. Let $c_{2}+2 c_{3}+c_{4}=11$. Obviously $c_{1}=4$ and $u_{1}^{2}, u_{4}^{2} \in \mathfrak{T}$. If $c_{2}=2$ then Then we must have $c_{3}=4$. But then $c_{2}+2 c_{3}+c_{4} \geq 12$, since $c_{4} \geq 2$ by proposition 3 , which is a contradiction. So let $c_{2} \geq 3$. If $c_{2}=4$ then we must have $c_{3}=2$ and $c_{4}=3$, since $c_{2}+2 c_{3}+c_{4}=11$ and $c_{4} \geq 2$. So we have $4 c_{1}+3 c_{2}+c_{3} \geq 27$. If $c_{2}=3$ Then obviously $4 c_{1}+3 c_{2}+c_{3} \geq 27$, since $c_{3} \geq 2$ by proposition 3 . This completes the proof. $\diamond$

Lemma $134 c_{1}+3 c_{2}+c_{3} \geq 26$. Similarly, $4 c_{m}+3 c_{m-1}+c_{m-2} \geq 26$.

Proof. It is easy to see that $c_{1}=4$ and $u_{1}^{2}, u_{4}^{2} \in \mathfrak{T}$. If $c_{2} \geq 3$ then obviously $4 c_{1}+3 c_{2}+c_{3} \geq 27$ by proposition 3 . Let $c_{2}=2$. This forces $c_{3}=4$ and we have $4 c_{1}+3 c_{2}+c_{3} \geq 26$. This completes the proof. $\diamond$

Lemma 14 There is no $i$ such that $c_{j}+2 c_{j+1}+c_{j+2}=11$ for $i \leq j \leq i+4$.

Proof. Let there exists an $i$ such that $c_{j}+2 c_{j+1}+c_{j+2}=11$ for $i \leq j \leq i+4$. We consider three cases.

case $1 \quad c_{i}=4$. This forces $c_{i+1}=2$ and $c_{i+2}=3$, since $c_{i}+2 c_{i+1}+c_{i+2}=11$. If $u_{1}^{i+1}, u_{4}^{i+1} \in \mathfrak{T}$ or if $u_{2}^{i+1}, u_{3}^{i+1} \in \mathfrak{T}$ then we must have $c_{i+2}=4$ which is a contradiction. Now, consider two subcases.

subcase $1 u_{1}^{i+1}, u_{3}^{i+1} \in \mathfrak{T}$. This forces $u_{1}^{i+2}, u_{3}^{i+2}, u_{4}^{i+2} \in \mathfrak{T}$. Since $c_{i+1}+$ $2 c_{i+2}+c_{i+3}=11$ we must have $c_{i+3}=3$ and $u_{1}^{i+3}, u_{2}^{i+3}, u_{4}^{i+3} \in \mathfrak{T}$. Now by assumption we must have $c_{i+4}=2$ and $u_{2}^{i+4}, u_{4}^{i+4} \in \mathfrak{T}$. This implies $c_{i+5}=4$. But by proposition 3 we have $c_{i+4}+2 c_{i+5}+c_{i+6} \geq 12$ which is a contradiction. subcase $2 u_{2}^{i+1}, u_{4}^{i+1} \in \mathfrak{T}$. An argument similar to that described in subcase 1 leads to a contradiction.

case $2 \quad c_{i}=3$. By assumption we must have $c_{i+1}=3$ and $c_{i+2}=2$. This implies $c_{i+3}=4$ since $c_{i+1}+2 c_{i+2}+c_{i+3}=11$. This forces $c_{i+2}+2 c_{i+3}+c_{i+4} \geq 12$ by proposition 3 , which is a contradiction.

case $3 c_{i}=2$. Then we have $2 c_{i+1}+c_{i+2}=9$ which forces $c_{i+1}=c_{i+2}=3$. Now we must have $c_{i+3}=2$ and $c_{i+4}=4$. But then we have $c_{i+3}+2 c_{i+4}+c_{i+5} \geq$ 12 by proposition 3 , which is a contradiction. This completes the proof. $\diamond$

Theorem $15 \gamma_{\times 3}\left(P_{4} \times P_{m}\right)=\left\lceil\frac{11 m+\left\lfloor\frac{m-4}{5}\right\rfloor+9}{4}\right\rceil$. 
Proof. We have

$$
\begin{aligned}
4|\mathfrak{T}| & =\sum_{i=1}^{m} 4 c_{i} \\
& =\left(4 c_{1}+3 c_{2}+c_{3}\right)+\sum_{i=2}^{m-3}\left(c_{i}+2 c_{i+1}+c_{i+2}\right)+\left(4 c_{m}+3 c_{m-1}+c_{m-2}\right) .
\end{aligned}
$$

We consider two cases.

case $1 \quad c_{2}+2 c_{3}+c_{4}=11$. Then by lemma 12 , we have $4 c_{1}+3 c_{2}+c_{3} \geq 27$. Now we have

$$
\begin{aligned}
4|\mathfrak{T}| & \geq 53+\sum_{i=2}^{m-3}\left(c_{i}+2 c_{i+1}+c_{i+2}\right) \\
& \geq 53+12\left\lfloor\frac{n-4}{5}\right\rfloor+11\left(4\left\lfloor\frac{n-4}{5}\right\rfloor\right)+11\left(n-4-5\left\lfloor\frac{n-4}{5}\right\rfloor\right) \\
& =11 n+\left\lfloor\frac{n-4}{5}\right\rfloor+9
\end{aligned}
$$

Since By lemma 14 at most one of the consecutive five terms $c_{i}+2 c_{i+1}+c_{i+2}$ is greater than 11 and the other is greater than 10 .

case $2 c_{2}+2 c_{3}+c_{4}=12$. Then it is easy to see that

$$
\begin{aligned}
4|\mathfrak{T}| & \geq 26+\sum_{i=2}^{m-3}\left(c_{i}+2 c_{i+1}+c_{i+2}\right)+\left(4 c_{n}+3 c_{n-1}+c_{n-2}\right) \\
& \geq 26+\left(12\left\lfloor\frac{n-4}{5}\right\rfloor+11\left(4\left\lfloor\frac{n-4}{5}\right\rfloor\right)+11\left(n-4-5\left\lfloor\frac{n-4}{5}\right\rfloor\right)+27\right) \\
& =11 n+\left\lfloor\frac{n-4}{5}\right\rfloor+9
\end{aligned}
$$

Thus $|\mathfrak{T}| \geq\left\lceil\frac{11 m+\left\lfloor\frac{m-4}{5}\right\rfloor+9}{4}\right\rceil$. 
Now we define a 3-tuple dominating set of order $\left\lceil\frac{9 m-\left\lceil\frac{m-2}{5}\right\rceil+4}{4}\right\rceil$. Let $m=5 s+r$, $s, r \in \mathbb{Z}$ and $0 \leq r<m$. Define

$$
\begin{aligned}
\mathfrak{T}= & \left\{u_{i}^{j} \mid 0 \leq i \leq 4, j=1, n, 5 k+3 \text { and } 0 \leq k \leq s-1\right\} \cup\left\{u_{1}^{2}, u_{4}^{2}\right\} \cup \\
& \left\{u_{1}^{5 k+4}, u_{3}^{5 k+4} \mid 0 \leq k \leq s-1\right\} \cup \\
& \left\{u_{1}^{5 k}, u_{3}^{5 k}, u_{4}^{5 k} \mid 1 \leq k \leq s-1\right\} \cup \\
& \left\{u_{1}^{5 k+1}, u_{2}^{5 k+1}, u_{4}^{5 k+1} \mid 1 \leq k \leq s-1\right\} \cup \\
& \left\{u_{2}^{5 k+2}, u_{4}^{5 k+2} \mid 1 \leq k \leq s-1\right\} \cup \\
& \begin{cases}\left\{u_{1}^{m-1}, u_{4}^{m-1}\right\} & \text { if } s \geq 1, r=0 \\
\left\{u_{1}^{m-2}, u_{3}^{m-2}, u_{1}^{m-1}, u_{3}^{m-1}, u_{4}^{m-1}\right\} & \text { if } s \geq 1, r=1 \\
\left\{u_{1}^{m-3}, u_{4}^{m-3}, u_{1}^{m-1}, u_{4}^{m-1} u_{i}^{m-2} \mid 1 \leq i \leq 4\right\} & \text { if } \quad r=2 \\
\left\{u_{1}^{m-4}, u_{3}^{m-4}, u_{1}^{m-3}, u_{3}^{m-3}, u_{4}^{m-3}\right\} \cup & \text { if } r=3 \\
\left\{u_{1}^{m-2}, u_{2}^{m-2}, u_{4}^{m-2}, u_{2}^{m-1}, u_{4}^{m-1}\right\} & \text { if } r=4 . \\
\left\{u_{i}^{m-5}, u_{i}^{m-3}, u_{i}^{m-1} \mid i=1,4\right\} \cup & \\
\left\{u_{i}^{m-4}, u_{i}^{m-2} \mid 1 \leq i \leq 4\right\} & \end{cases}
\end{aligned}
$$

It is easy to see that in each case $\mathfrak{T}$ is a 3 -tuple dominating set in $P_{4} \times P_{m}$ with $\left\lceil\frac{11 m+\left\lfloor\frac{m-4}{5}\right\rfloor+9}{4}\right\rceil$ vertices. This completes the proof. $\diamond$

\section{3 perfect 3 -tuple dominating sets}

In this section we focus on perfect 3-tuple dominating sets for grids, cylinders and torus. In subsection 3.1 we completely characterize all grids that possess a perfect 3-tuple dominating sets and also specify the structure of the existing perfect 3-tuple dominating sets. In subsection 3.2 we determined which cylinders contain a perfect 3-tuple dominating sets and characterize the structure of their perfect 3-tuple dominating sets. Subsection 3.3 is devoted to determine all torus which contain a perfect 3 -tuple dominating sets and to characterize the structure of their perfect 3 -tuple dominating sets.

\section{1 perfect 3-tuple dominating sets for grids}

In this subsection we characterize all grids $P_{n} \times P_{m}$ that have perfect 3 -tuple dominating set and determine the construction of their existing perfect 3 -tuple dominating sets. Throughout this section $\mathfrak{T}$ is a perfect 3 -tuple dominating set in $P_{n} \times P_{m}$ when it does exist. We assume that the vertices of the $i$ th copy of $P_{n}$ in $P_{n} \times P_{m}$ are $u_{1}^{i}, u_{2}^{i}, u_{3}^{i}, \ldots, u_{n}^{i}$ for $i=1,2, \ldots, m$. (See Figure 1.) We also assume that $\mathfrak{T}$ has precisely $c_{i}$ vertices in the $i$ th copy of $P_{n}$ in $P_{n} \times P_{m}$. 
Lemma $16 \quad P_{2} \times P_{m}$ has a perfect 3 -tuple dominating set if and only if $m=2$.

Proof. Suppose that $\mathfrak{T}$ is a perfect 3 -tuple dominating set of $P_{2} \times P_{m}$. Since $u_{1}^{1}$ and $u_{2}^{1}$ must be dominated by three vertices of $\mathfrak{T}$ we must have $u_{1}^{1}, u_{2}^{1}, u_{1}^{2}, u_{2}^{2} \in$ $\mathfrak{T}$. If $m=2$ then $\mathfrak{T}$ is a perfect 3 -tuple dominating set of $P_{2} \times P_{2}$. Let $m \geq 3$. Then we must have $c_{3}=0$. But then $u_{1}^{3}$ is dominated by at most two vertices of $\mathfrak{T}$ which is a contradiction. This completes the proof. $\diamond$

Lemma 17 Let $m \geq 3$. Then $P_{3} \times P_{m}$ has a perfect 3 -tuple dominating set if and only if $m=4$.

Proof. Let $\mathfrak{T}$ be a perfect 3-tuple dominating set in $P_{3} \times P_{m}$. Since $u_{1}^{1}$ and $u_{3}^{1}$ must be dominated by three vertices of $\mathfrak{T}$ we must have $u_{1}^{1}, u_{2}^{1}, u_{3}^{1}, u_{1}^{2}, u_{3}^{2} \in \mathfrak{T}$. This forces $u_{2}^{2}, u_{2}^{3} \notin \mathfrak{T}$ which implies $u_{1}^{3}, u_{3}^{3}, u_{1}^{4}, u_{2}^{4}, u_{3}^{4} \in \mathfrak{T}$. If $m=4$ then obviously $\mathfrak{T}$ is a perfect 3 -tuple dominating set of $P_{3} \times P_{4}$. Let $m \geq 5$. Then we must have $c_{5}=0$. But then $u_{1}^{5}$ is dominated by at most two vertices of $\mathfrak{T}$ which is a contradiction. This completes the proof. $\diamond$

Lemma 18 If $m, n \geq 4$ then $P_{n} \times P_{m}$ does not has a perfect 3-tuple dominating set.

Proof. Let $\mathfrak{T}$ be a perfect 3 -tuple dominating set in $P_{n} \times P_{m}$. It is clear that $u_{1}^{1}, u_{2}^{1}, u_{1}^{2} \in \mathfrak{T}$. If $u_{3}^{1} \notin \mathfrak{T}$ then we must have $u_{2}^{2}, u_{3}^{2} \in \mathfrak{T}$ which is a contradiction. Let $u_{3}^{1} \in \mathfrak{T}$. This forces $u_{2}^{2} \notin \mathfrak{T}$ and $u_{1}^{3} \in \mathfrak{T}$. We consider two cases.

case $1 \quad u_{3}^{2} \in \mathfrak{T}$. This forces $u_{4}^{1} \notin \mathfrak{T}$ which implies $u_{4}^{2} \in \mathfrak{T}$. This forces $u_{3}^{3} \notin \mathfrak{T}$. But then $u_{3}^{2}$ is dominated by at most two vertices of $\mathfrak{T}$ which is a contradiction. case $2 \quad u_{3}^{2} \notin \mathfrak{T}$. This implies $u_{2}^{3} \in \mathfrak{T}$. In a similar vein, we obtain a contradiction. This completes the proof. $\diamond$

Now we state the main result of this subsection.

Theorem $19 P_{n} \times P_{m}$ has a perfect 3-tuple dominating set if and only if

$$
\left\{\begin{array}{l}
n=m=2 \\
n=3 \text { and } m=4 .
\end{array}\right.
$$

\section{2 perfect 3-tuple dominating sets for cylinders}

In this subsection we characterize cylinders which contain a perfect 3-tuple dominating set and determined the structure of their 3-tuple dominating sets. We assume that $u_{1}^{i}, u_{2}^{i}, \ldots, u_{n}^{i}$ are the vertices of the $i$ th copy of $C_{n}$ in $C_{n} \times P_{m}$ for $i=1,2, \ldots, m$. We also assume that $\mathfrak{T}$ is a 3 -tuple dominating set in $C_{n} \times P_{n}$ and has precisely $c_{i}$ vertices in the $i$ th copy of $C_{n}$ in $C_{n} \times P_{m}$. Throughout this section $n \geq 3$.

The proof of the following result is straightforward and we leave it for the reader. 
Lemma $20 C_{n} \times P_{1}$ has a perfect 3-tuple dominating set for each $n \geq 3$.

Lemma $21 \quad C_{n} \times P_{2}$ has a perfect 3-tuple dominating set if and only if $n$ is multiply of 4 .

Proof. Suppose that $\mathfrak{T}$ is a perfect 3-tuple dominating set of $C_{n} \times P_{2}$. Without loss of generality we can assume $u_{1}^{1} \notin \mathfrak{T}$. This forces $u_{1}^{2}, u_{3}^{1}, u_{2}^{1}, u_{2}^{2} \in \mathfrak{T}$. Since $\mathfrak{T}$ is a perfect 3 -tuple dominating set we must have $u_{3}^{2} \notin \mathfrak{T}$. With a similar argument we have $u_{1}^{5} \notin \mathfrak{T}$ and $u_{1}^{4} \in \mathfrak{T}$. Now, an induction argument shows that $u_{1}^{4 k+1} \notin \mathfrak{T}$ and $u_{1}^{4 k+2}, u_{1}^{4 k+3}, u_{1}^{4 k+4} \in \mathfrak{T}$ for each $k \geq 0$. This implies $4 \mid n$.

Conversely, Let $4 \mid n$. Define

$$
\mathfrak{T}=\left\{u_{1}^{4 k+2}, u_{1}^{4 k+3}, u_{1}^{4 k+4}, u_{2}^{4 k+1}, u_{2}^{4 k+2}, u_{2}^{4 k+4} \mid 0 \leq k \leq \frac{n}{4}-1\right\} .
$$

It is easy to see that $\mathfrak{T}$ is a perfect 3 -tuple dominating set in $C_{n} \times P_{2}$. This completes the proof. $\diamond$

Lemma $22 C_{n} \times P_{m}$ has no perfect 3-tuple dominating set for any two integers $n, m \geq 3$.

Proof. Let $\mathfrak{T}$ be a perfect 3-tuple dominating set in $C_{n} \times P_{m}$. Without loss of generality, we can assume $u_{1}^{1} \notin \mathfrak{T}$. This forces $u_{1}^{n}, u_{1}^{2}, u_{2}^{n}, u_{2}^{1}, u_{2}^{2} \in \mathfrak{T}$ which implies $u_{3}^{n}, u_{3}^{1}, u_{3}^{2} \notin \mathfrak{T}$. But then $u_{3}^{1}$ is dominated by at most two vertices of $\mathfrak{T}$ which is a contradiction. This completes the proof. $\diamond$

Now we state the main result of this subsection.

Theorem $23 C_{n} \times P_{m}$ has a perfect 3-tuple dominating set if and only if

$$
\left\{\begin{array}{l}
n \geq 3 \text { and } m=1 \\
n \equiv 0(\bmod 4) \text { and } m=2 .
\end{array}\right.
$$

\section{3 perfect 3-tuple dominating sets for torus}

In this subsection we characterize all torus $C_{n} \times C_{m}, m, n \geq 3$, that have perfect 3 -tuple dominating set and determine the construction of their existing perfect 3 -tuple dominating sets. We assume that $u_{1}^{i}, u_{2}^{i}, \ldots, u_{n}^{i}$ are the vertices of the $i$ th copy of $C_{n}$ in $C_{n} \times C_{m}$ for $i=1,2, \ldots, m$. We also assume that $\mathfrak{T}$ has precisely $c_{i}$ vertices in the $i$ th copy of $C_{n}$ in $C_{n} \times C_{m}$. Throughout this section $n, m \geq 3$.

Lemma $24 C_{3} \times C_{m}$ has no perfect 3-tuple dominating set for any positive integer $m$. 
Proof. Let $\mathfrak{T}$ be a perfect 3 -tuple dominating set in $C_{3} \times C_{m}$. It is easy to see that $c_{i} \geq 1$ for each $i$. We claim that $c_{i}=2$ for $i=1,2, \ldots, n$. Let $c_{i} \neq 2$ for some $i$. If $c_{i}=3$ then we must $c_{i+1}=0$. But then the vertices in $i$ th copy of $C_{3}$ is dominated by at most two vertices of $\mathfrak{T}$ which is a contradiction. If $c_{i}=1$ then we must have $c_{i+1}=3$ which leads to a contradiction. Thus $c_{i}=2$ for each $i$. Without loss of generality we can assume $u_{1}^{1}, u_{2}^{1} \in \mathfrak{T}$. This implies $u_{3}^{n}$ or $u_{3}^{2} \in \mathfrak{T}$. Without loss of generality we can assume $u_{3}^{2} \in \mathfrak{T}$. Since $c_{2}=2$ we must have $u_{2}^{1}$ or $u_{2}^{2} \in \mathfrak{T}$. But then we must have $c_{3}=1$ which is a contradiction. This completes the proof. $\diamond$

Lemma $25 C_{4} \times C_{m}$ has no perfect 3-tuple dominating set for any integer $m \geq 4$.

Proof. By Lemma 24 we can assume $m \geq 4$. Let $\mathfrak{T}$ be a perfect double dominating set in $C_{4} \times C_{m}$. It is easy to see that $2 \leq c_{i} \leq 3$ for each $i$. If $c_{i}=2$ for some $i$ then obviously $c_{i+1}=c_{i-1}=1$ which is a contradiction. So $c_{i}=3$ for each $i$. Without loss of generality we can assume $u_{1}^{1}, u_{2}^{1}, u_{3}^{1} \in \mathfrak{T}$. Since $u_{4}^{1}$ must be dominated by exactly three vertices of $\mathfrak{T}$ we must have $u_{4}^{n}$ or $u_{4}^{2} \in \mathfrak{T}$. Without loss of generality we can assume $u_{4}^{2} \in \mathfrak{T}$. This forces $u_{4}^{n}, u_{2}^{n} \notin \mathfrak{T}$ and $c_{n} \leq 2$ which is a contradiction. This completes the proof. $\diamond$

Lemma 26 Let $m, n \geq 5$ and $\mathfrak{T}$ be a perfect 3 -tuple dominating set for $C_{n} \times$ $C_{m}$. Then there is no $i, j$ such that $u_{i}^{j}, u_{i}^{j+1}, u_{i}^{j+2}, u_{i}^{j+3} \in \mathfrak{T}$ or $u_{i}^{j}, u_{i+1}^{j}, u_{i+2}^{j}$, $u_{i+3}^{j} \in \mathfrak{T}$.

Proof. Let $u_{i}^{j}, u_{i}^{j+1}, u_{i}^{j+2}, u_{i}^{j+3} \in \mathfrak{T}$ for some $i, j$. This forces $u_{i-1}^{j+1}, u_{i-1}^{j+2}, u_{i+1}^{j+1}$, $u_{i+1}^{j+2} \notin \mathfrak{T}$ which implies $u_{i-1}^{j-1}, u_{i+1}^{j-1} \in \mathfrak{T}$. Then $u_{i}^{j}$ is dominated by at least four vertices of $\mathfrak{T}$ which is a contradiction. $\diamond$

Lemma 27 Let $m, n \geq 5$ and $\mathfrak{T}$ be a perfect 3 -tuple dominating set for $C_{n} \times$ $C_{m}$. Then there is no $i, j$ such that $u_{i}^{j}, u_{i}^{j+1}, u_{i+1}^{j}, u_{i+1}^{j+1} \in \mathfrak{T}$.

Proof. Let $u_{i}^{j}, u_{i}^{j+1}, u_{i+1}^{j}, u_{i+1}^{j+1} \in \mathfrak{T}$ for some $i, j$. This forces $u_{i}^{j+2}, u_{i}^{j-1}, u_{i+1}^{j+2}$, $u_{i+1}^{j-1}, u_{i+2}^{j}, u_{i+2}^{j+1}, u_{i-1}^{j}, u_{i-1}^{j+1} \notin \mathfrak{T}$. Then we must have $u_{i-2}^{j-1}, u_{i-2}^{j}, u_{i-2}^{j+1}, u_{i-2}^{j+2} \in \mathfrak{T}$. This leads to a contradiction by lemma $26 . \diamond$

Lemma 28 Let $m, n \geq 5$ and $\mathfrak{T}$ be a perfect 3 -tuple dominating set for $C_{n} \times C_{m}$. Then there is no $i, j$ such that $u_{i}^{j}, u_{i}^{j+1}, u_{i}^{j+2}, u_{i+1}^{j}, u_{i+1}^{j+2} \in \mathfrak{T}$ or $u_{i}^{j}, u_{i}^{j+1}, u_{i}^{j+2}, u_{i-1}^{j}$,

$u_{i-1}^{j+2} \in \mathfrak{T}$. Similarly, there is no $i, j$ such that $u_{i}^{j}, u_{i+1}^{j}, u_{i+2}^{j}, u_{i}^{j+1}, u_{i+2}^{j+1} \in \mathfrak{T}$ or $u_{i}^{j}, u_{i+1}^{j}, u_{i+2}^{j}, u_{i}^{j-1}, u_{i+2}^{j-1} \in \mathfrak{T}$.

Proof. Let $u_{i}^{j}, u_{i}^{j+1}, u_{i}^{j+2}, u_{i+1}^{j}, u_{i+1}^{j+2} \in \mathfrak{T}$ for some $i, j$. This forces $u_{i-1}^{j}, u_{i-1}^{j+1}$, $u_{i-1}^{j+2} \notin \mathfrak{T}$. But then $u_{i-1}^{j+1}$ is dominated by at most two vertices of $\mathfrak{T}$ which is a contradiction. $\diamond$ 
Lemma 29 Let $m, n \geq 5$. If $C_{n} \times C_{m}$ has a perfect 3 -tuple dominating set then both $n$ and $m$ are multiples of 5 .

Proof. Let $\mathfrak{T}$ be a perfect 3-tuple dominating set in $C_{n} \times C_{m}$. Without loss of generality, we can assume $u_{4}^{1}, u_{4}^{2} \in \mathfrak{T}$. By lemma 26 we must have $u_{4}^{m}$ or $u_{4}^{3} \notin \mathfrak{T}$. Consider three cases.

Case $1 \quad u_{4}^{m} \notin \mathfrak{T}$ and $u_{4}^{3} \in \mathfrak{T}$. This implies $u_{3}^{2}, u_{5}^{2} \notin \mathfrak{T}$. By lemma 28 we must have $u_{3}^{1}, u_{5}^{3} \in \mathfrak{T}$ or $u_{3}^{3}, u_{5}^{1} \in \mathfrak{T}$. without loss of generality we can assume $u_{3}^{1}, u_{5}^{3} \in \mathfrak{T}$. This forces $u_{3}^{3}, u_{5}^{1}, u_{4}^{4} \notin \mathfrak{T}$ and $u_{2}^{2}, u_{2}^{3}, u_{2}^{4}, u_{3}^{4}, u_{3}^{5}, u_{6}^{2}, u_{6}^{1}, u_{6}^{m}, u_{5}^{m} \in \mathfrak{T}$. Thus, $u_{2}^{1}, u_{6}^{3} \notin \mathfrak{T}$ by lemma 28. This forces $u_{1}^{2}, u_{5}^{4}, u_{7}^{2} \in \mathfrak{T}$. This forces $u_{1}^{3}, u_{1}^{4}, u_{2}^{5}, u_{4}^{5}, u_{7}^{m}, u_{7}^{1} \notin \mathfrak{T}$ and $u_{1}^{5}, u_{1}^{6}, u_{3}^{6}, u_{4}^{6}, u_{5}^{5}, u_{8}^{1}, u_{8}^{m} \in \mathfrak{T}$. But then $u_{2}^{6}, u_{3}^{7}, u_{5}^{6}$, $u_{6}^{4}, u_{8}^{2} \notin \mathfrak{T}$ and $u_{2}^{7}, u_{4}^{7}, u_{6}^{5}, u_{7}^{3}, u_{7}^{4}, u_{9}^{1} \in \mathfrak{T}$. This implies $u_{3}^{8}, u_{7}^{5}, u_{8}^{3} \notin \mathfrak{T}$ and $u_{9}^{2}, u_{9}^{3}, u_{8}^{4}, u_{6}^{6}, u_{4}^{8}, u_{3}^{9}, u_{2}^{8} \in \mathfrak{T}$. Now, we must have $u_{9}^{4}, u_{5}^{7} \notin \mathfrak{T}$ and $u_{10}^{3}, u_{5}^{8} \in \mathfrak{T}$. So far we have proved if $u_{4}^{1}, u_{4}^{2}, u_{4}^{3}, u_{3}^{1}, u_{5}^{3} \in \mathfrak{T}$ then $u_{4}^{6}, u_{4}^{7}, u_{4}^{8}, u_{6}^{1}, u_{8}^{3} \in \mathfrak{T}$ and $u_{9}^{1}, u_{9}^{2}, u_{9}^{3}, u_{8}^{1}, u_{10}^{3} \in \mathfrak{T}$. A simple induction argument shows that

$$
u_{5 k+3}^{1}, u_{5 k+4}^{1} \in \mathfrak{T} \text { and } u_{4}^{5 k+1}, u_{4}^{5 k+2}, u_{4}^{5 k+3} \in \mathfrak{T}
$$

for every non-negative integer $k$. (Note that the superscript $5 k+a$ in $u_{i}^{5 k+a}$ is the unique integer $r \in\{1,2, \ldots, m\}$ such that $5 k+a \equiv r(\bmod m)$ and the subscript $5 k+b$ in $u_{5 k+b}^{j}$ is the unique integer $s \in\{1,2, \ldots, n\}$ such that $5 k+b \equiv s(\bmod n)$.) Now since $u_{1}^{5 k+3} \in \mathfrak{T}$ for every non-negative integer $k$ it follows that 5 divides $m$, otherwise $u_{1}^{i} \in \mathfrak{T}$ for every $i=1,2, \ldots, m$ which is impossible. Similarly, 5 divides $n$ since $u_{5 k+1}^{4} \in \mathfrak{T}$ for every non-negative integer $k$.

Case $2 \quad u_{4}^{m} \in \mathfrak{T}$ and $u_{4}^{3} \notin \mathfrak{T}$. In a similar fashion with case 1 we can prove that 5 divides $n$ and $m$.

Case $3 u_{4}^{m}, u_{4}^{3} \notin \mathfrak{T}$. This forces $u_{3}^{1}, u_{5}^{2} \in \mathfrak{T}$ or $u_{3}^{2}, u_{5}^{1} \in \mathfrak{T}$ by lemma 27 . Without loss of generality we can assume $u_{3}^{1}, u_{5}^{2} \in \mathfrak{T}$. This forces $u_{3}^{3}, u_{5}^{1} \notin \mathfrak{T}$ and $u_{3}^{3}, u_{3}^{4}, u_{2}^{3} \in \mathfrak{T}$. This implies $u_{2}^{2} \notin \mathfrak{T}$ and $u_{2}^{1}, u_{1}^{2} \in \mathfrak{T}$. This forces $u_{3}^{m} \notin \mathfrak{T}$ and $u_{2}^{m} \in \mathfrak{T}$. Now by relabeling the vertices of $C_{n} \times C_{m}$ this case can be converted to Case 1 . So 5 divides $m$ and $n$. $\diamond$

Lemma 30 Let 5 divides $m$ and $n$. Then

$$
\mathfrak{T}=\left\{u_{5 k+2 j-1}^{j}, u_{5 k+2 j}^{j}, u_{k+2 j+1}^{j}, \mid 0 \leq j \leq m, 0 \leq k<\frac{n}{5}-1\right\}
$$

where addition in subscribe is taken module $n$, is a perfect 3 -tuple dominating set in $C_{n} \times C_{m}$.

Proof. It is straightforward to check that each vertex of $\mathfrak{T}$ is dominated by exactly two vertex of $\mathfrak{T}$.

Now let $u_{i}^{j} \in V \backslash \mathfrak{T}$. Then $i \neq 5 k+2 j-1,5 k+2 j, 5 k+2 j+1$ for each nonnegative integer $k$, by definition of $\mathfrak{T}$. It is easy to see that 


$$
\begin{array}{lll}
\text { if } i=5 k+2 j+2 & \text { then } & u_{i}^{j} \in N\left[u_{i}^{j-1}\right] \cap N\left[u_{i}^{j+1}\right] \cap N\left[u_{i-1}^{j}\right] \text {; } \\
\text { if } i=5 k+2 j+3 & \text { then } & u_{i}^{j} \in N\left[u_{i}^{j-1}\right] \cap N\left[u_{i}^{j+1}\right] \cap N\left[u_{i+1}^{j}\right] .
\end{array}
$$

It is also easy to see that in each case $u_{i}^{j}$ is not dominated by another vertex of $\mathfrak{T}$. So $u_{i}^{j}$ is dominated by exactly three vertices of $\mathfrak{T}$. Thus, $\mathfrak{T}$ is a perfect 3-tuple dominating set in $C_{n} \times C_{m}$. $\diamond$

Now we state the main result of this subsection.

Theorem 31 Let $n, m \geq 3$. Then $C_{n} \times C_{m}$ has a perfect 3 -tuple dominating set if and only if 5 divides both $m$ and $n$.

\section{References}

[1] J. Borges and I.J. Dejter, On perfect dominating sets in hypercubes and their complements, Journal of Combinatorial Mathematics and Combinatorial Computing 20 (1996), 161-173.

[2] T. Y. Chang, W.E. Clark and E. O. Hare, Domination numbers of complete grid graphs, I, Ars Combinatoria 38 (1994), 97-111.

[3] G. Chartrand, H. Gavlas, R. C. Vandell and F. Harary, The forcing domination number of a graph, Journal of Combinatorial Mathematics and Combinatorial Computing 25 (1997), 161-174.

[4] F. Harary, Graph Theory, Addison-Wesley, Reading, MA, 1969.

[5] F. Harary, and T. W. Haynes, Double domination in graphs, Ars Combinatoria 55 (2000), 201-213.

[6] T. W. Haynes, S. T. Hedetniemi and P. J. Slater, Fundamentals of domination in graphs. Marcel Dekker, Inc., New York, 1998.

[7] M. Livingston and Q.F. Stout, Perfect dominating sets, Proceedings of the Twenty-first Southeastern Conference on Combinatorics, Graph Theory, and Computing (Boca Raton, FL, 1990). Congressus Numererantium 79 (1990), 187-203.

[8] A. Khodkar and S.M. Sheikholeslami, The forcing domination numbers of some graphs, (submitted).

[9] A. Khodkar and S.M. Sheikholeslami, On perfect double dominating set, (submitted).

[10] P. J. Slater, Maximum facility location, J. Res. Nat. Bur. Standards 79B (1975) 107-115. 
[11] D. Van Wieren, M. Livingston and Q.F. Stout, Perfect dominating sets on cube-connected cycles, Proceedings of the Twenty-fourth Southeastern International Conference on Combinatorics, Graph Theory, and Computing (Boca Raton, FL, 1993). Congressus Numererantium 97 (1993), 51-70.

Received: October 14, 2005 